\title{
Membrane fatty acid composition of rat skeletal muscle is most responsive to the balance of dietary $n-3$ and $n-6$ PUFA
}

\author{
Sarah K. Abbott ${ }^{1,2 *}$, Paul L. Else ${ }^{1,3}$ and A. J. Hulbert ${ }^{1,2}$ \\ ${ }^{1}$ Metabolic Research Centre, University of Wollongong, Wollongong, NSW 2522, Australia \\ ${ }^{2}$ School of Biological Sciences, University of Wollongong, Northfields Avenue, Wollongong, NSW 2522, Australia \\ ${ }^{3}$ School of Health Sciences, University of Wollongong, Wollongong, NSW 2522, Australia
}

(Received 22 April 2008 - Revised 27 August 2009 - Accepted 28 August 2009 - First published online 13 October 2009)

The present study quantifies the relationships between diet fatty acid profile and fatty acid composition of rat skeletal muscle phospholipids. Young adult male Sprague-Dawley rats were fed, for 8 weeks, on one of twelve moderate-fat diets (25\% of total energy) differing only in fatty acid profile. SFA content ranged from $8-88 \%$ of total fatty acids, MUFA $6-65 \%$, total PUFA $4-81 \%, n-6$ PUFA 3-70\% and $n-3$ PUFA $1-70 \%$. Diet PUFA included only essential fatty acids $18: 2 n-6$ and $18: 3 n-3$. The balance between $n-3$ and $n-6$ PUFA (PUFA balance) in the diet ranged from $1: 99$ to $86: 14 \%$ n-3 PUFA:n-6 PUFA. The slope of muscle phospholipid composition plotted against diet composition quantifies the response of muscle membrane composition to dietary fat (0, no response; 1 , complete conformity with diet). The resulting slopes were 0.02 (SFA), 0.10 (PUFA), 0.11 (MUFA), 0.14 ( $n$-3 PUFA) and 0.23 (n-6 PUFA). The response to PUFA balance was biphasic with a slope of 0.98 below $10 \%$ diet PUFA balance and 0.16 above $10 \%$. Thus, low diet PUFA balance has greater influence on muscle composition than 18-carbon $n-3$ or $n$-6 PUFA individually. Equations provided may allow prediction of muscle composition for other diet studies. Diet PUFA balance dramatically affects muscle $20: 4 n-6$ and $22: 6 n-3$. This may have significant implications for some disease states in human subjects.

n-3: n-6: Obesity: Arachidonic acid: DHA

Higher animals can synthesise both SFA and MUFA from non-lipid sources, but are unable to synthesise de novo both $n-6$ and $n-3$ PUFA. They are also incapable of interconverting these two classes of polyunsaturates and consequently both $n-6$ and $n-3$ PUFA are separate essential dietary components. Indeed, in the food chain, both $n-6$ and n-3 PUFA are synthesized de novo by plants as 18-carbon fatty acids and are further elongated and desaturated by animals to produce longer chain $n-6$ and $n-3$ PUFA. The dietary importance of $n-6$ and $n-3$ PUFA derives from the fact that they are both essential constituents of membrane lipids of higher animals. The PUFA composition of cell membrane bilayers has important effects on the functionality of the great variety of proteins (e.g. hormone receptors, neuroreceptors, membrane pumps) that are embedded in cellular membranes. Also, as constituents of membrane lipids, PUFA are the precursors of important signalling molecules (e.g. eicosanoids and endocannabinoids).

It has been long known that the fatty acid composition of the diet can influence membrane fatty acid composition. Most studies compare a very small number of diets (normally only two diets) and to our knowledge there are no studies that have mathematically quantified the relationship between diet and membrane composition. Studies in animals show that membrane fatty acid composition is influenced via the process of constant membrane remodelling, whereby fatty acids that constitute membrane lipids are being constantly removed and replaced by a complex series of membrane-bound enzymes. Acyltransferases, an important group of enzymes involved in this process, are highly selective for polyunsaturates but do not discriminate well between $n-6$ PUFA and $n-3$ PUFA $^{(1)}$. Consequently, membrane fatty acid composition is likely, a priori, very responsive to the balance between $n-6$ and $n$ - 3 PUFA in the diet.

To quantify the relationship between the fatty acid composition of the diet and membrane lipids, one recent analysis ${ }^{(2)}$ has applied the 'conformer/regulator' paradigm ${ }^{(3)}$ to literature data for the laboratory rat. It showed that, while membrane composition is relatively unresponsive to the SFA and MUFA content of the diet, it is most responsive to the ratio of $n-3$ PUFA to $n-6$ PUFA. This conclusion was, however, based on the comparison of four to five diets of which only one of the diets was high in $n$-3 PUFA (see Fig. 2 of Hulbert et al. ${ }^{(2)}$ ) and consequently lacked robustness.

The present study was designed to overcome this problem. It compares the effect of twelve isoenergetic diets, identical except for fatty acid composition, on the fatty acid composition of muscle phospholipids of the rat. All diets had a moderate fat content ( $25 \%$ of total energy). To quantify the diet-membrane relationship accurately, the twelve diets

Abbreviations: B, beef dripping; C, coconut oil; \% en, percentage energy; F, flaxseed oil; O, olive oil; S, safflower oil. 
were designed to supply a wide range of relative content of SFA, MUFA, $n-6$ PUFA and $n-3$ PUFA. Only 18-carbon $n-3$ and $n-6$ PUFA were provided as these are the dominant PUFA in the normal diet of rats. Differences in lipid metabolism exist between rodents and human subjects, so caution must be taken when extrapolating results from an animal model to human conditions. These differences will be discussed later.

Unlike the earlier analysis that used the ' $n$-3 PUFA: $n-6$ PUFA' ratio $^{(2)}$, here we use in its place what we have called 'PUFA balance' (defined as ' $n-3$ PUFA as percentage of total PUFA') to analyse the interaction between these two types of PUFA in the diet on membrane composition. PUFA balance is analogous to a proportion (or a ratio where the two values sum to $100 \%)$. We discuss the advantages of this approach later in this paper. In the present study, the diet $n$-3 PUFA: $n-6$ PUFA ranged from 1:99 (Diet 1) to 86:14 (Diet 12) and was found to be the most influential on membrane fatty acid composition. This may have significant implications for some disease states in human subjects.

\section{Materials and methods}

\section{Animals}

All experiments were approved by the University of Wollongong Animal Ethics Committee and were conducted in conformity with the Public Health Service policy on Humane Care and Use of Laboratory Animals. Eight-week-old male Sprague-Dawley rats (Gore Hill Research Laboratories, Sydney) were housed individually under a $12: 12 \mathrm{~h}$ light:dark schedule. The room was maintained at $23^{\circ} \mathrm{C}$ and $40 \%$ relative humidity. Before dietary manipulation, all rats were fed ad libitum on commercial rodent diet (Y.S. Feeds, Young, Australia), the composition of which was $13 \%$ en (percentage energy) fat, $22 \%$ en protein and $65 \%$ en carbohydrate.

Rats were randomly assigned to either the initial group ( $n$ 6) or one of the twelve dietary groups ( $n 6$ per group). Rats initially weighed 295.7 (SE 2.5) $\mathrm{g}(n$ 78) and following diet treatment weighed 528.2 (SE 7.5) g ( $n$ 72). An initial group of rats were euthanised at 8 weeks of age to give an indication of muscle fatty acid composition before dietary intervention. Following diet treatment, rats were euthanised via intraperitoneal injection $(200 \mathrm{mg} / \mathrm{kg}$ body mass) of Lethabarb (sodium pentobarbitone, Virbac, Peakhurst, NSW, Australia). The skeletal muscle (medial gastrocnemius) was immediately removed, frozen and stored at $-80^{\circ} \mathrm{C}$ until analysis.

\section{Diets}

Rats were fed ad libitum for 8 weeks on complete diets differing only in fatty acid profile (Tables 1 and 2). The average fatty acyl chain length was 17.0 (SE 0.2) carbons for all diets. No 20-carbon or 22-carbon PUFA were included in the diets. Diets were designed to cover a wide range of SFA, MUFA, $n-3$ PUFA, $n-6$ PUFA and PUFA balance, which was achieved by mixing various proportions of the following oils: flaxseed oil (F; Nutralife Hi-Omega, Moorebank, NSW, Australia); safflower oil (S; Healthy Life Cold Pressed, Milperra, NSW, Australia); olive oil (O; Lupi Cold Pressed Extra Virgin, Gosford, NSW, Australia); coconut oil
Table 1. Composition of experimental diets (see text for details)

\begin{tabular}{lc}
\hline Ingredients & Amount $(\mathrm{g} / \mathrm{kg})$ \\
\hline Oil mix & 120 \\
Sucrose & 82 \\
Maize starch & 493 \\
Casein & 155 \\
Fibre & 50 \\
Mineral mix & 40 \\
Vitamin mix & 10 \\
Gelatin & 50 \\
Energy contribution (\% total energy) & \\
$\quad$ Carbohydrate & 55 \\
Protein & 20 \\
Fat & 25 \\
\hline
\end{tabular}

(C; Aclara Health Organic Gourmet Cold Pressed Extra Virgin, Mount Crosby, QLD, Australia); beef dripping (B; Allowrie Prime, Rowville, VIC, Australia).

The diet fatty acid composition was achieved by mixing $\mathrm{F}$, $\mathrm{S}, \mathrm{O}, \mathrm{C}$ and $\mathrm{B}$ in the following percentages (percentage of total fat content): Diet 1 (61\% F and 39\% O); Diet $2(1 \% \mathrm{~F}, 42 \%$ $\mathrm{S}, 2 \% \mathrm{O}$ and $55 \% \mathrm{~B})$; Diet $3(8 \% \mathrm{~F}$ and $92 \% \mathrm{~S})$; Diet $4(1 \%$ F, $1 \% \mathrm{~S}, 6 \% \mathrm{O}$ and $92 \% \mathrm{~B})$; Diet $5(10 \% \mathrm{~F}$ and $90 \% \mathrm{O})$; Diet $6(41 \% \mathrm{~F}, 29 \% \mathrm{~S}$ and $30 \% \mathrm{O})$; Diet 7 (42\% F, $33 \%$ $\mathrm{S}$ and $25 \% \mathrm{C})$; Diet $8(5 \% \mathrm{~F}, 2 \% \mathrm{~S}$ and $93 \% \mathrm{C})$; Diet 9 (35\% F, $27 \% \mathrm{~S}$ and $38 \% \mathrm{~B})$; Diet 10 (78\% F and $22 \% \mathrm{~S})$; Diet $11(30 \% \mathrm{~F}$ and $70 \% \mathrm{C})$; Diet 12 (98\% F and $2 \% \mathrm{C})$.

Other diet constituents were sucrose (CSR White Sugar, Yarraville, VIC, Australia), maize starch (Fielders Wheaten Cornflour, PFD Food Services, Wollongong, NSW, Australia), casein (Rogers \& Co. Edible Rennet, Brighton, QLD, Australia), fibre (Vitacel Wheat Fibre Type WF600, generously donated by Nutrimonde, Mascot, Australia), mineral and vitamin mix (MP Biomedicals: AIN-93M and AIN-93-VX, respectively, Seven Hills, NSW, Australia) and gelatin (Gelita Gelatine Edible powder, Krio Krush, Kingsgrove, NSW, Australia).

\section{Fatty acid analysis}

All solvents used were HPLC grade (Crown Scientific, Moorebank, NSW, Australia) and contained 0.01\% w/v butylated hydroxytoluene (Sigma Aldrich, Sydney, NSW, Australia). Total lipids were extracted from the skeletal muscle using chloroform-methanol (2:1, v:v) and glass/glass homogenisers as previously described ${ }^{(4)}$. Phospholipids were separated from total lipids using Sep-Pak ${ }^{\circledR}$ Classic Silica Cartridge (Waters, Rydalmere, NSW, Australia). Phospholipids were transmethylated $^{(5)}$ and fatty acid methyl esters were measured by GC (Shimadzu GC-17A, Rydalmere, NSW, Australia) using a Varian WCOT fused silica column $(50 \mathrm{~m} \times 0.25 \mathrm{~mm}$ internal diameter, CP7419, Sydney, New South Wales, Australia) with the following temperature program: $150^{\circ} \mathrm{C}$ initial temperature; $17.5^{\circ} \mathrm{C} / \mathrm{min}$ to $170^{\circ} \mathrm{C} ; 0.5^{\circ} \mathrm{C} / \mathrm{min}$ to $178^{\circ} \mathrm{C}$; $15^{\circ} \mathrm{C} / \mathrm{min}$ to $222^{\circ} \mathrm{C} ; 2^{\circ} \mathrm{C} / \mathrm{min}$ to $232^{\circ} \mathrm{C}$. Individual fatty acids were identified by comparison with an external standard (FAME Mix C4-C24; Sigma Aldrich, Sydney, Australia) and then expressed as mole percentage of total fatty acids. 


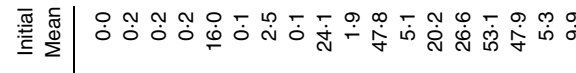 \\ Statistical analyses}

|山

$\simeq$

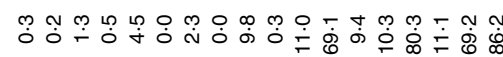

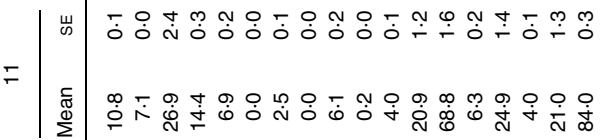

|山

우

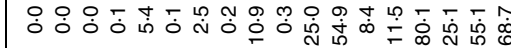

|山

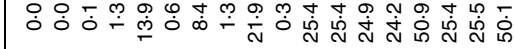

Ш

$\infty$

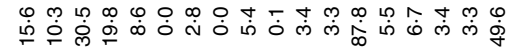

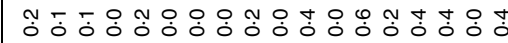

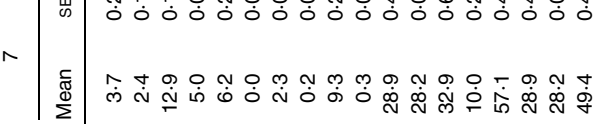

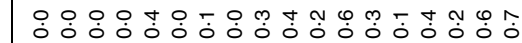

• |

س

เ

ர்

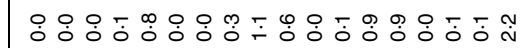

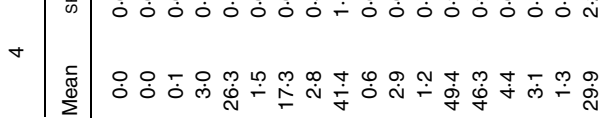

山ّ

$\infty$

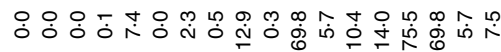

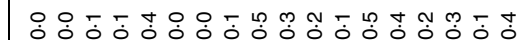

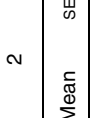

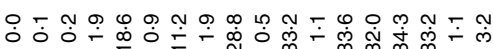

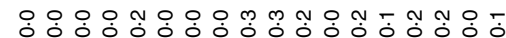

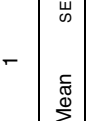

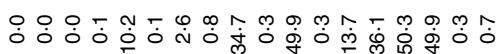

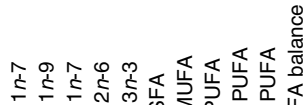

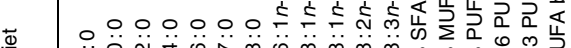

Data analysis was performed using JMP 5.1 (Statistical Analysis System Institute Inc., Cary, NC, USA). All results are expressed as means with their standard errors with $P<0.005$ set as the level of significance. Data were tested for normality using the Shapiro-Wilk $W$ test and homogeneity of variance using the O'Brien and Brown-Forsythe tests. Data not normally distributed were compared using non-parametric Wilcoxon/Kruskal-Wallis tests and any data showing unequal variance were compared using a Welch ANOVA. A one-way ANOVA was completed with diet as the independent variable and each fatty acid as the dependent variable. Means were then compared using the Tukey-Kramer honestly significant difference test. Linear regression was employed for plots comparing diet and membrane fatty acid composition followed by a one-way ANOVA as an indication of fit. Data for individual rats were used for all linear regression analyses ( $n 72$ data points); however, only the mean and standard error for each diet group are plotted in each figure. Data for the initial group of rats are also plotted in all figures (shown as solid squares), but was not included in any linear regression analysis.

\section{Results}

Fatty acid composition of the skeletal muscle phospholipids is presented in Table 3. Significant differences were evident between the diet groups for all fatty acids, except 16:0 and 18:0 (Table 3). In the muscle phospholipids, $22: 6 n-3$ accounted for the majority of the $n-3$ PUFA content, while $18: 2 n-6$ and $20: 4 n-6$ provided the highest proportions in the total $n$-6 PUFA composition (Table 3).

The skeletal muscle results were analysed using the classic conformer/regulator concept ${ }^{(3)}$, with the muscle fatty acid composition plotted against the dietary fatty acid composition (Fig. 1). The slope of each relationship represents how responsive membrane composition is to diet composition. A slope of one indicates membrane composition conforms completely to diet fatty acid composition, while a value of zero indicates a membrane regulating a constant fatty acid composition irrespective of diet composition. The results of the linear regression analysis for each figure are provided in Table 4, which also includes some regression analyses of relationships not plotted in the figures.

The muscle composition was unresponsive to large changes in diet SFA with a slope of 0.02 (Fig. 1(a)). This also means that membrane composition is similarly unresponsive to the total unsaturated fatty acid content in the diet (as these are co-dependant variables). Muscle membrane composition was more responsive to diet MUFA content with a responsivity of 0.11 (Fig. 1(a)). Although muscle response to diet total PUFA was $0 \cdot 10$ (Fig. 1(a)), the response to the two separate classes of PUFA was much greater. The slope for the $n-3$ PUFA relationship was $0 \cdot 14$ (Table 4), and the greatest overall slope was for the response of muscle to diet $n$ - 6 PUFA with a value of 0.23 (Table 4 ).

The diet-membrane relationship with the highest slope was found for the PUFA balance (Fig. 1(b)) with a value of 0.98 at diet PUFA balance $<10 \%$. This value indicates that muscle PUFA balance changed by $98 \%$ for a $100 \%$ change in diet PUFA balance. The PUFA balance data do not seem to 
Table 3. Fatty acid composition (percentage of total fatty acid) of skeletal muscle phospholipids from rats fed experimental and initial diets (Mean values with their standard errors, $n 6$ )

\begin{tabular}{|c|c|c|c|c|c|c|c|c|c|c|c|c|c|c|c|c|c|c|c|c|c|c|c|c|c|c|}
\hline \multirow{2}{*}{ Diet } & \multicolumn{2}{|c|}{1} & \multicolumn{2}{|r|}{2} & \multicolumn{2}{|c|}{3} & \multicolumn{2}{|c|}{4} & \multicolumn{2}{|c|}{5} & \multicolumn{2}{|r|}{6} & \multicolumn{2}{|r|}{7} & \multicolumn{2}{|r|}{8} & \multicolumn{2}{|r|}{9} & \multicolumn{2}{|c|}{10} & \multicolumn{2}{|r|}{11} & \multicolumn{2}{|c|}{12} & \multicolumn{2}{|c|}{ Initial } \\
\hline & Mean & $\mathrm{SE}$ & Mean & $\mathrm{SE}$ & Mean & $\mathrm{SE}$ & Mean & $\mathrm{SE}$ & Mean & $\mathrm{SE}$ & Mean & $\mathrm{SE}$ & Mean & SE & Mean & SE & Mean & $\mathrm{SE}$ & Mean & SE & Mean & SE & Mean & $\mathrm{SE}$ & Mean & $\mathrm{SE}$ \\
\hline $14: 0^{*}$ & 0.1 & $0.0^{\mathrm{d}}$ & 0.1 & $0.1^{\mathrm{d}}$ & 0.1 & $0.0^{d}$ & 0.2 & $0.1^{\mathrm{c}, \mathrm{d}}$ & 0.1 & $0.1^{d}$ & 0.1 & $0.1^{\mathrm{d}}$ & 0.5 & $0.1^{\mathrm{b}, \mathrm{c}}$ & 1.0 & $0.1^{\mathrm{a}}$ & 0.1 & $0.1^{\mathrm{d}}$ & 0.0 & $0.0^{\mathrm{d}}$ & 0.7 & $0.1^{a, b}$ & 0.1 & $0.0^{d}$ & 0.1 & $0.1^{d}$ \\
\hline $16: 0$ & 22.9 & 1.1 & 24.7 & 0.1 & $23 \cdot 3$ & 0.9 & $23 \cdot 2$ & 0.9 & 23.8 & 0.9 & 24.0 & 0.7 & 23.9 & 0.6 & $25 \cdot 1$ & 0.7 & 23.7 & 0.8 & 23.0 & 0.9 & 24.7 & 0.8 & 21.8 & 1.0 & $25 \cdot 1$ & 0.2 \\
\hline $18: 0$ & $15 \cdot 3$ & 0.6 & $15 \cdot 2$ & 0.3 & 15.6 & 0.6 & 15.1 & 0.5 & 13.9 & 0.3 & 14.4 & 0.6 & 14.8 & 0.4 & 13.9 & 0.3 & 15.4 & 0.5 & 14.9 & 0.7 & 14.3 & 0.3 & $16 \cdot 1$ & 0.4 & 14.1 & 0.3 \\
\hline $16: 1 n-7^{\star}$ & 0.5 & $0.1^{\mathrm{d}}$ & 0.6 & $0.0^{c, d}$ & 0.4 & $0.0^{\mathrm{d}}$ & 1.0 & $0.1^{\mathrm{b}}$ & 0.5 & $0.0^{\mathrm{d}}$ & 0.5 & $0.1^{\mathrm{d}}$ & 0.7 & $0.1^{\mathrm{b}, \mathrm{c}, \mathrm{d}}$ & 1.6 & $0.2^{\mathrm{a}}$ & 0.6 & $0.1^{c, d}$ & 0.6 & $0.1^{\mathrm{b}, \mathrm{c}, \mathrm{d}}$ & 1.0 & $0.1^{\mathrm{b}, \mathrm{c}}$ & 0.5 & $0.0^{\mathrm{d}}$ & 0.7 & $0.0^{\mathrm{b}, \mathrm{c}, \mathrm{d}}$ \\
\hline $18: 1 n-9^{*}$ & 6.6 & $0.5^{\mathrm{b}, \mathrm{c}}$ & 4.8 & $0.2^{c, d, e, f}$ & 3.4 & $0.1^{\dagger}$ & 9.8 & $0.5^{\mathrm{a}}$ & 11.0 & $0.7^{\mathrm{a}}$ & $6 \cdot 3$ & $0 \cdot 2^{\mathrm{b}, c, \mathrm{~d}}$ & 4.2 & $0 \cdot 3^{\mathrm{e}, \mathrm{f}}$ & 6.8 & $0.6^{\mathrm{b}}$ & 5.5 & $0.5^{\mathrm{b}, \mathrm{c}, \mathrm{d}, \mathrm{e}}$ & 4.2 & $0.3^{\mathrm{e}, \mathrm{f}}$ & 5.6 & $0.2^{\mathrm{b}, \mathrm{c}, \mathrm{d}, \mathrm{e}}$ & 4.7 & $0 \cdot 2^{\mathrm{d}, \mathrm{e}, \mathrm{f}}$ & 6.1 & $0.1^{b, c, d, e}$ \\
\hline $18: 1 n-7^{\star}$ & 2.6 & $0.1^{\mathrm{b}, \mathrm{c}}$ & 2.3 & $0.0^{\mathrm{c}, \mathrm{d}, \mathrm{e}}$ & $2 \cdot 1$ & $0.0^{e, f}$ & 2.7 & $0.1^{\mathrm{b}}$ & 3.0 & $0.1^{\mathrm{a}}$ & 2.3 & $0.0^{\mathrm{c}, \mathrm{d}, \mathrm{e}}$ & 2.2 & $0.1^{\mathrm{d}, \mathrm{e}, \mathrm{f}}$ & 3.0 & $0.1^{\mathrm{a}}$ & $2 \cdot 2$ & $0.1^{\mathrm{d}, \mathrm{e}, \mathrm{f}}$ & 2.0 & $0.1^{e, f}$ & $\begin{array}{l}2.0 \\
2.5\end{array}$ & $0 \cdot 1^{b, c, d}$ & $\begin{array}{l}4.1 \\
1.9\end{array}$ & $\begin{array}{l}0.1^{\dagger} \\
0.1^{2}\end{array}$ & $\begin{array}{l}.1 \\
3.2\end{array}$ & $0.1^{\mathrm{a}}$ \\
\hline $18: 2 n-6^{*}$ & 17.7 & $1 \cdot 7^{\mathrm{c}, \mathrm{d}, \mathrm{de}}$ & 14.9 & $0.3^{\mathrm{e}}$ & 22.3 & $1 \cdot 2^{\mathrm{a}, \mathrm{b}, \mathrm{c}}$ & 15.0 & $0.6^{\mathrm{e}}$ & $\begin{array}{r}14.2 \\
\end{array}$ & $1.0^{\mathrm{e}}$ & 20.3 & $1 \cdot 1^{a, b, c, d}$ & 21.6 & $0.6^{\mathrm{a}, \mathrm{b}, \mathrm{b}} \mathrm{c}$ & $16 \cdot 3$ & $0.6^{\mathrm{d}, \mathrm{e}}$ & 18.6 & $1 \cdot 4^{\mathrm{b}, \mathrm{c}, \mathrm{d}, \mathrm{e},}$ & 24.3 & $1.0^{\mathrm{a}}$ & 18.0 & $1 \cdot 3^{\mathrm{c}, \mathrm{d}, \mathrm{e}}$ & 23.3 & $1 \cdot 2^{\mathrm{a}, \mathrm{b}}$ & $\begin{array}{l}3.2 \\
17.8\end{array}$ & $0 \cdot 3^{\mathrm{c}, \mathrm{d}, \mathrm{e}}$ \\
\hline $20: 3 n-6^{*}$ & 0.5 & $0.0^{\mathrm{d}, \mathrm{e}}$ & 0.6 & $0.0^{\mathrm{c}, \mathrm{d}, \mathrm{e}}$ & 0.5 & $0.0^{\mathrm{e}}$ & 0.9 & $0.0^{\mathrm{a}}$ & 0.7 & $0.0^{\mathrm{b}, \mathrm{c}}$ & 0.6 & $0.0^{c, d, e}$ & 0.7 & $0.0^{\mathrm{cd}}$ & 0.9 & $0.1^{\mathrm{a}, \mathrm{b}}$ & 0.7 & $0.0^{\mathrm{c}, \mathrm{d}}$ & 0.6 & $0.0^{\mathrm{c}, \mathrm{d}, \mathrm{e}}$ & 0.7 & $0.0^{c}$ & 0.6 & $0.0^{\mathrm{c}, \mathrm{d}, \mathrm{e}}$ & 1.0 & $0.0^{\mathrm{a}}$ \\
\hline $20: 4 n-6^{*}$ & 19.6 & $1 \cdot 2^{\mathrm{a}, \mathrm{b}}$ & 20.4 & $0.2^{\mathrm{a}}$ & 16.4 & $0.7^{b, c}$ & 13.8 & $0 \cdot 6^{\mathrm{c}, \mathrm{d}, \mathrm{e}}$ & 12.1 & $0.7^{\mathrm{d}, \mathrm{e}, \mathrm{f}}$ & 11.5 & $0.9^{e, f, g}$ & 10.1 & $0.5^{\mathrm{fg}, \mathrm{h}}$ & 9.7 & $0 \cdot 8^{\mathrm{f}, \mathrm{g}, \mathrm{h}, \mathrm{i}}$ & 11.9 & $0 \cdot 6^{\mathrm{de}, \mathrm{e}, \mathrm{f}}$ & 8.2 & $0.4^{\mathrm{g}, \mathrm{h}, \mathrm{i}}$ & 7.4 & $0.6^{\mathrm{h}, \mathrm{i}}$ & 6.5 & $0.6^{i}$ & 15.0 & $0.4^{\mathrm{c}, \mathrm{d}}$ \\
\hline $22: 4 n-6^{*}$ & 1.1 & $0.1^{\mathrm{a}}$ & 0.8 & $0.1^{\mathrm{b}}$ & 0.6 & $0.1^{\mathrm{c}}$ & 0.3 & $0.0^{\mathrm{d}, \mathrm{e}}$ & 0.1 & $0.0^{f}$ & 0.0 & $0.0^{f}$ & 0.1 & $0.1^{\dagger}$ & 0.2 & $0.0^{\mathrm{e}, \mathrm{f}}$ & 0.1 & $0.0^{f}$ & 0.0 & $0.0^{f}$ & 0.1 & $0.0^{f}$ & 0.0 & $0.0^{4}$ & 0.6 & $0.0^{\mathrm{c}, \mathrm{d}}$ \\
\hline $22: 5 n-6^{\star}$ & 3.2 & $0.1^{\mathrm{a}}$ & 1.9 & $0.3^{\mathrm{b}}$ & 0.7 & $0.1^{c}$ & 0.5 & $0.1^{\mathrm{c}}$ & 0.0 & $0.0^{\mathrm{d}}$ & 0.0 & $0.0^{\mathrm{d}}$ & 0.0 & $0.0^{d}$ & 0.3 & $0.1^{\mathrm{c}, \mathrm{d}}$ & 0.0 & $0.0^{\mathrm{d}}$ & 0.0 & $0.0^{\mathrm{d}}$ & 0.0 & $0.0^{\mathrm{d}}$ & 0.0 & $0.0^{\mathrm{d}}$ & 0.5 & $0.0^{c}$ \\
\hline $18: 3 n-3^{*}$ & 0.1 & $0.0^{\mathrm{d}}$ & 0.0 & $0.0^{\mathrm{d}}$ & 0.2 & $0.1^{\mathrm{d}}$ & 0.0 & $0.0^{\mathrm{d}}$ & 0.4 & $0.0^{d}$ & 1.3 & $0.1^{\circ}$ & 1.7 & $0.2^{\mathrm{c}}$ & 0.3 & $0.1^{d}$ & 1.0 & $0.1^{c}$ & 3.2 & $0.2^{\mathrm{b}}$ & 1.7 & $0.3^{\mathrm{c}}$ & 4.6 & $0.3^{\mathrm{a}}$ & 0.1 & $0.1^{\mathrm{d}}$ \\
\hline $20: 5 n-3^{*}$ & 0.0 & $0.0^{f}$ & 0.0 & $0.0^{4}$ & 0.0 & $0.0^{4}$ & 0.4 & $0.0^{e, f}$ & 0.7 & $0.1^{\mathrm{d}, \mathrm{e}}$ & 0.8 & $0.1^{\mathrm{d}, \mathrm{e}}$ & 1.0 & $0.1^{c, d}$ & 0.8 & $0.1^{\mathrm{d}, \mathrm{e}}$ & 0.8 & $0.1^{\mathrm{d}, \mathrm{e}}$ & 1.4 & $0.0^{\mathrm{b}, \mathrm{c}}$ & 1.9 & $0.3^{\mathrm{a}, \mathrm{b}}$ & 2.2 & $0.2^{\mathrm{a}}$ & 0.4 & $0.1^{e, f}$ \\
\hline $22: 5 n-3^{*}$ & 1.0 & $0.1^{\mathrm{h}}$ & 2.0 & $0.1^{g, h}$ & 2.0 & $0.1^{\mathrm{g}, \mathrm{h}}$ & 2.5 & $0.1^{\mathrm{f}, \mathrm{g}}$ & 3.4 & $0.2^{e, f}$ & 4.1 & $0 \cdot 3^{\mathrm{c}, \mathrm{d}, \mathrm{e}}$ & 4.7 & $0.2^{b, c, d}$ & $\begin{array}{l}3.0 \\
3.8\end{array}$ & $0.2^{\mathrm{d}, \mathrm{e}}$ & 4.8 & $0 \cdot 3^{\mathrm{b}, \mathrm{c}, \mathrm{d}}$ & 5.2 & $0 \cdot 4^{\mathrm{a}, \mathrm{b}, \mathrm{c}}$ & 6.1 & $0.5^{\mathrm{a}}$ & 5.5 & $0.4^{\mathrm{a}, \mathrm{b}}$ & $\begin{array}{l}2.4 \\
2.5\end{array}$ & $0.1^{\mathrm{t}, \mathrm{g}}$ \\
\hline $22: 6 n-3^{*}$ & 8.1 & $0.3^{f}$ & 10.5 & $0.5^{\mathrm{e}}$ & 11.4 & $0.5^{\mathrm{d}, \mathrm{e}}$ & 13.1 & $0 \cdot 3^{\mathrm{b}, \mathrm{c}, \mathrm{d}}$ & 15.4 & $0.7^{\mathrm{a}, \mathrm{b}}$ & 13.0 & $0.4^{\mathrm{c}, \mathrm{d}}$ & 12.9 & $0.4^{\mathrm{c}, \mathrm{d}, \mathrm{e}}$ & 15.6 & $0.3^{\mathrm{a}}$ & 13.1 & $0 \cdot 6^{\mathrm{b}, \mathrm{c}, \mathrm{d}}$ & 11.5 & $0.6^{\mathrm{d}, \mathrm{e}}$ & 14.5 & $0.8^{\mathrm{a}, \mathrm{b}, \mathrm{c}}$ & 10.9 & $0.3^{\mathrm{d}, \mathrm{e}}$ & $\begin{array}{r}11.9\end{array}$ & $0 \cdot 2^{\mathrm{d}, \mathrm{e}}$ \\
\hline$\%$ SFA & 38.8 & $0.9^{\mathrm{a}}$ & 41.0 & $0.6^{\mathrm{a}}$ & 39.5 & $0.8^{\mathrm{a}}$ & 39.5 & $0.7^{\mathrm{a}}$ & 38.3 & $0.8^{\mathrm{a}}$ & 39.1 & $0.8^{\mathrm{a}}$ & 39.7 & $0.4^{\mathrm{a}}$ & 40.5 & $0.8^{\mathrm{a}}$ & 40.5 & $0.6^{\mathrm{a}}$ & 38.3 & $0.4^{\mathrm{a}}$ & 40.3 & $0.7^{\mathrm{a}}$ & 38.6 & $1.0^{\mathrm{a}}$ & 40.0 & $0.3^{\mathrm{a}}$ \\
\hline$\%$ MUFA $^{*}$ & $\begin{array}{r}0.0 \\
9.8\end{array}$ & $0 \cdot 5^{\mathrm{b}, \mathrm{c}}$ & 7.8 & $0 \cdot 2^{\mathrm{c}, \mathrm{d}, \mathrm{e}}$ & $\begin{array}{r}6.0 \\
6.0\end{array}$ & $0.2^{\mathrm{e}}$ & $\begin{array}{l}14.0 \\
14.0\end{array}$ & $0.6^{\mathrm{a}}$ & $\begin{array}{l}0.0 \\
14.8\end{array}$ & $0.7^{\mathrm{a}}$ & $\begin{array}{r}9.1 \\
9.2\end{array}$ & $0.2^{\mathrm{b}, \mathrm{c}, \mathrm{d}}$ & $\begin{array}{r}39.7 \\
7.3\end{array}$ & $\begin{array}{l}0.4^{\mathrm{d}}, \mathrm{e} \\
0 . \mathbf{n}^{2}\end{array}$ & $\begin{array}{l}40.5 \\
11.5\end{array}$ & $\begin{array}{l}0.8^{\mathrm{b}} \\
0.9^{\mathrm{s}}\end{array}$ & $\begin{array}{r}4.5 \\
8.4\end{array}$ & $\begin{array}{l}0.6^{c}, \mathrm{cos} \\
0.7^{2}\end{array}$ & $\begin{array}{r}8.3 \\
6.9\end{array}$ & $\begin{array}{l}0.4^{\mathrm{d}}, \mathrm{d} \\
0.4^{2}\end{array}$ & $\begin{array}{r}40.3 \\
9.2\end{array}$ & 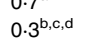 & $\begin{array}{r}38.6 \\
7.3\end{array}$ & $\begin{array}{l}1.0 \\
0.3^{\mathrm{d}, \mathrm{e}}\end{array}$ & $\begin{array}{l}40.0 \\
10.1\end{array}$ & $\begin{array}{l}0.3^{b}, \mathrm{~b} \\
0 . x^{2}\end{array}$ \\
\hline$\%$ PUFA $^{*}$ & $\begin{array}{r}51.4 \\
51\end{array}$ & $0.5^{b, c, d}$ & 51.3 & $0.5^{\mathrm{c}, \mathrm{d}}$ & 54.5 & $0.8^{a, b}$ & 46.6 & $0.4^{9}$ & $\begin{array}{l}46.9 \\
46.9\end{array}$ & $0.8^{f, g}$ & $\begin{array}{l}51.7 \\
51.7\end{array}$ & $0.6^{\mathrm{a}, \mathrm{b}, \mathrm{c}, \mathrm{d}}$ & $\begin{array}{r}52.9 \\
\end{array}$ & $0 \cdot 4^{\mathrm{a}, \mathrm{b}, \mathrm{c}, \mathrm{d}}$ & $\begin{array}{l}17.9 \\
\end{array}$ & $0.8^{\mathrm{e}, \mathrm{fg}}$ & 51.1 & $0.9^{\mathrm{c}, \mathrm{d}}$ & 54.8 & $0.5^{\mathrm{a}}$ & $\begin{array}{r}50.5 \\
50.5\end{array}$ & $0.6^{\mathrm{d}, \mathrm{e}}$ & 54.2 & $0.9^{\mathrm{a}, \mathrm{b}, \mathrm{c}}$ & 49.9 & $0.4^{\mathrm{d}, \mathrm{e}, \mathrm{f}}$ \\
\hline$n-6$ PUFA $^{*}$ & $42 \cdot 3$ & $0.6^{\mathrm{a}}$ & 38.7 & $0.1^{\mathrm{b}}$ & 40.8 & $0.8^{\mathrm{a}, \mathrm{b}}$ & 30.5 & $0.4^{\mathrm{d}, \mathrm{e}}$ & 27.1 & $0 \cdot 6^{6, g}$ & 32.4 & $0.5^{\mathrm{c}, \mathrm{d}}$ & 32.6 & $0.5^{\mathrm{c}, \mathrm{d}}$ & 27.4 & $0.9^{\text {e,fg }}$ & 31.4 & $1.0^{\mathrm{d}}$ & 33.2 & $0.7^{\mathrm{c}, \mathrm{d}}$ & $26 \cdot 1$ & $1 \cdot 2^{\mathrm{g}}$ & 30.5 & $0.7^{\mathrm{d}, \mathrm{e}, \mathrm{f}}$ & 35.0 & $0.5^{\mathrm{c}}$ \\
\hline$n-3$ PUFA $^{*}$ & 9.1 & $0.4^{4}$ & 12.6 & $0.6^{\mathrm{e}}$ & 13.7 & $0.4^{\mathrm{d}, \mathrm{e}}$ & 16.0 & $0.3^{d}$ & 19.8 & $0.6^{\circ}$ & 19.2 & $0.3^{\mathrm{c}}$ & 20.3 & $0.5^{\mathrm{c}}$ & 20.5 & $0.2^{\mathrm{c}}$ & $\begin{array}{l}31.4 \\
19.7\end{array}$ & $0.7^{\circ}$ & $\begin{array}{l}30.2 \\
21.6\end{array}$ & $0.5^{\mathrm{b}, \mathrm{c}}$ & 24.4 & $1.1^{\mathrm{a}}$ & $\begin{array}{l}3.5 \\
23.7\end{array}$ & $0.7^{\mathrm{a}, \mathrm{b}}$ & $\begin{array}{l}30.0 \\
14.9\end{array}$ & $\begin{array}{l}0.5 \\
0.2^{\mathrm{d}, \mathrm{e}}\end{array}$ \\
\hline PUFA & 17.7 & $0.8^{i}$ & 24.5 & $0.8^{h}$ & $25 \cdot 1$ & $0.8^{g, h}$ & 34.4 & $0.7^{e, f}$ & 42.2 & $0.8^{b, c}$ & 37.2 & $0.6^{\mathrm{d}, \mathrm{e}}$ & 38.4 & $0.9^{\mathrm{c}, \mathrm{d}, \mathrm{e}}$ & 42.9 & $0.9^{\mathrm{b}, \mathrm{c}}$ & 38.7 & $1 \cdot 4^{\mathrm{d}, \mathrm{d}, \mathrm{e}}$ & 39.5 & $1 \cdot 0^{b, c, d}$ & $\begin{array}{l}2 \cdot 4 \\
48 \cdot 3\end{array}$ & $2 \cdot 1^{\mathrm{a}}$ & $\begin{array}{l}20.1 \\
43.7\end{array}$ & $0.9^{\mathrm{a}, \mathrm{b}}$ & 29.9 & $0 \cdot 6^{f, g}$ \\
\hline
\end{tabular}

a,b,c,d,e,t,g,h,i Mean values for each fatty acid without a common superscript in each row are significantly different $(P<0.0001)$. 
(a)

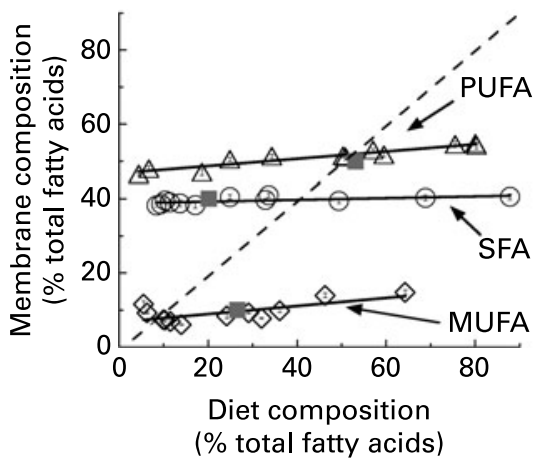

(b)

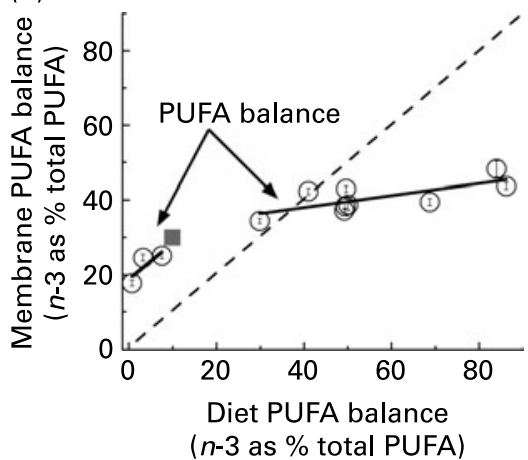

Fig. 1. Fatty acid profile from rat muscle phospholipids relative to fatty acid profile of diet. (a) SFA content; MUFA content; total PUFA content. (b) Relationship of PUFA balance ( $n-3$ PUFA as percentage of total PUFA) of rat muscle skeletal phospholipids with diet PUFA balance (two mathematical relationships applied to the data points). In each graph, represents value for initial rats, while $\Delta, \bigcirc, \diamond$ represent data from experimental diets. Error bars show means with their standard errors, and if they are not visible they are smaller than symbol. The dotted line in each graph indicates line of perfect diet-membrane conformity. See Table 4 for regression equations and statistical values.

follow a single linear function, and this is illustrated in Fig. 1(b) where the data points are plotted in a biphasic manner. This plot reveals that at diet PUFA balance, $<10 \%$ the muscle PUFA balance is drastically reduced with a slope of 0.98 (Fig. 1(b)). Above 10\%, however, the muscle PUFA balance displays a diet responsivity of $0 \cdot 16$ (Fig. 1(b)).

In the present experiment, both $n-6$ and $n$-3 PUFA were supplied in the diet as 18-carbon PUFA molecules; $18: 2 n-6$ and 18:3n-3, respectively (Table 2). Both $n-6$ and $n-3$ PUFA in muscle phospholipids included substantial amounts of more unsaturated, 20- and 22-carbon acyl chains (Table 3), with the $20: 4 n-6$ and $22: 6 n-3$ predominant longer-chain PUFA present. In Fig. 2, we have analysed the abundance of $20: 4 n-6$ and $22: 6 n-3$ in muscle phospholipids relative to (i) the amount of the respective 18-carbon precursor fatty acid in the diet (left-hand graphs) and (ii) the PUFA balance of the diet (right-hand graphs). The regression equations and statistical data for similar analysis of the other 20- and 22-carbon PUFA are provided in Table 4.

For muscle 20- and 22-carbon n-6 PUFA, 20:4n-6 predominated with only low levels of $22: 4 n-6$ and $22: 5 n-6$ present (Table 3). Muscle 20:4n-6 increased in response to diet 18:2n-6 (Fig. 2(a)), but decreased in response to the PUFA balance of the diet (Fig. 2(b)). Muscle 22:4n-6 content was very low $(0-1.1 \%)$ and showed only minor changes in response to both diet $18: 2 n-6$ content and

Table 4. Results for all linear regression analyses including relationships plotted in Figs. 1-2 and additional relationships (graphs not shown)

\begin{tabular}{|c|c|c|c|c|c|c|}
\hline Muscle composition $(y)$ & Diet composition $(x)$ & Equation & Slope standard error & $R$ & $P$ & Fig. \\
\hline SFA & SFA & $y=38.80+0.02 x$ & 0.008 & 0.31 & 0.0076 & 1(a) \\
\hline MUFA & MUFA & $y=6.73+0.11 x$ & 0.015 & 0.65 & $<0.0001^{*}$ & $1(\mathrm{a})$ \\
\hline PUFA & PUFA & $y=46.72+0.10 x$ & 0.008 & 0.83 & $<0.0001^{*}$ & 1(a) \\
\hline PUFA balance† & PUFA balance & $y_{1}=18.70+0.98 x$ & 0.233 & 0.72 & $0.0007^{\star}$ & 1 (b) \\
\hline PUFA balance $\ddagger$ & PUFA balance & $y_{2}=31.62+0.16 x$ & 0.028 & 0.62 & $<0.0001^{*}$ & $1(\mathrm{~b})$ \\
\hline $20: 4 n-6$ & $18: 2 n-6$ & $y=8.86+1.17 x$ & 0.189 & 0.59 & $<0.0001^{*}$ & 2(a) \\
\hline $20: 4 n-6$ & PUFA balance & $y=18.85-0.15 x$ & 0.008 & 0.91 & $<0.0001^{*}$ & 2 (b) \\
\hline $22: 6 n-3 \S$ & $18: 3 n-3$ & $y_{1}=9.03+3.66 x$ & 1.081 & 0.65 & $0.0037^{\star}$ & 2(c) \\
\hline $22: 6 n-3 \|$ & $18: 3 n-3$ & $y_{2}=14.89-0.49 x$ & 0.075 & 0.67 & $<0.0001^{*}$ & $2(c)$ \\
\hline $22: 6 n-3 \dagger$ & PUFA balance & $y_{1}=8.24+0.46 x$ & 0.104 & 0.75 & $0.0004^{*}$ & 2(d) \\
\hline $22: 6 n-3 \ddagger$ & PUFA balance & $y_{2}=15.19-0.03 x$ & 0.014 & 0.31 & 0.0213 & 2(d) \\
\hline$n-6$ PUFA & $n-6$ PUFA & $y=26.99+0.23 x$ & 0.017 & 0.86 & $<0.0001^{*}$ & \\
\hline $22: 4 n-6$ & $18: 2 n-6$ & $y=0.02+0.09 x$ & 0.015 & 0.59 & $<0.0001^{*}$ & \\
\hline $22: 4 n-6$ & PUFA balance & $y=0.78-0.01 x$ & 0.001 & 0.84 & $<0.0001^{*}$ & \\
\hline $22: 5 n-6$ & $18: 2 n-6$ & $y=-0.05+0.20 x$ & 0.043 & 0.49 & $<0.0001^{*}$ & \\
\hline $22: 5 n-6 \dagger$ & PUFA balance & $y_{1}=3.27-0.36 x$ & 0.037 & 0.92 & $<0.0001^{*}$ & \\
\hline $22: 5 n-6 \ddagger$ & PUFA balance & $y_{2}=0.35-0.00 x$ & 0.001 & 0.48 & $0.0002^{*}$ & \\
\hline$n-3$ PUFA & $n-3$ PUFA & $y=15.56+0.14 x$ & 0.020 & 0.64 & $<0.0001^{*}$ & \\
\hline $20: 5 n-3$ & $18: 3 n-3$ & $y=0.30+0.22 x$ & 0.022 & 0.76 & $<0.0001^{*}$ & \\
\hline $22: 5 n-3$ & $18: 3 n-3$ & $y=2.67+0.44 x$ & 0.055 & 0.69 & $<0.0001^{*}$ & \\
\hline $20: 5 n-3$ & PUFA balance & $y=-0.22+0.02 x$ & 0.001 & 0.91 & $<0.0001^{*}$ & \\
\hline $22: 5 n-3$ & PUFA balance & $y=1.45+0.05 x$ & 0.003 & 0.89 & $<0.0001^{*}$ & \\
\hline
\end{tabular}

All fatty acid compositions are expressed as percentage total fatty acids, except for $18: 2 n-6$ and $18: 3 n-3$, which are expressed as g/ $100 \mathrm{~g}$ diet, and PUFA balance, which is expressed as $n$-3 PUFA as \% total PUFA.

* The slope of the line of fit is significantly different from zero.

$\dagger$ Linear regression plotted below $10 \%$ diet PUFA balance.

¥Linear regression plotted above $10 \%$ diet PUFA balance.

$\S$ Linear regression plotted below $1 \mathrm{~g} / 100 \mathrm{~g}$ diet $18: 3 n-3$.

|| Linear regression plotted above $1 \mathrm{~g} / 100 \mathrm{~g}$ diet $18: 3 n-3$. 


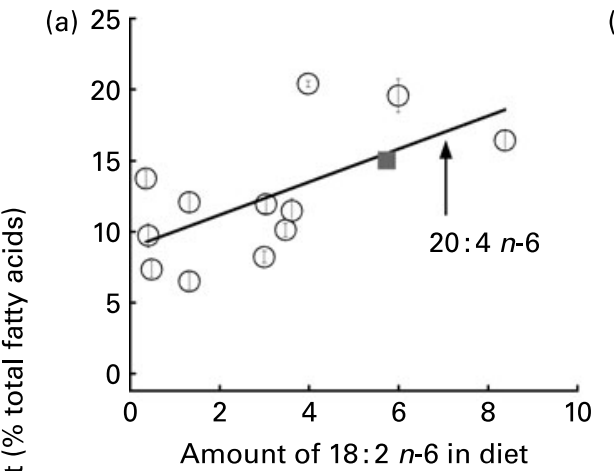

(g/100 g diet)

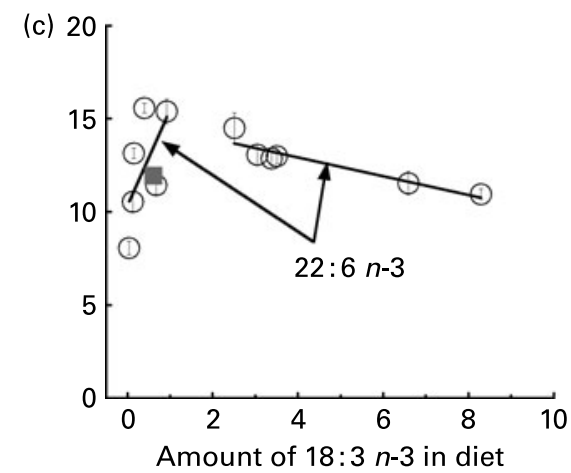

(g/100 g diet)

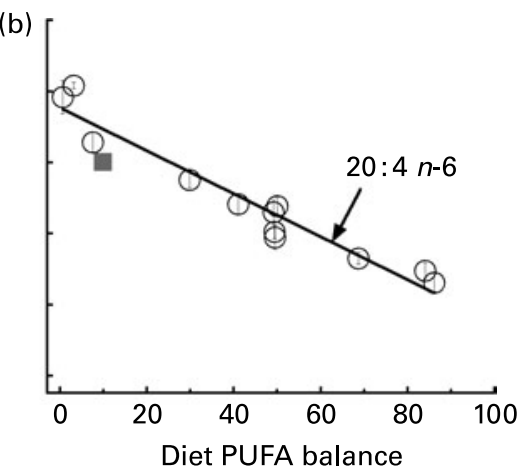

( $n-3$ as \% total PUFA)

(d)

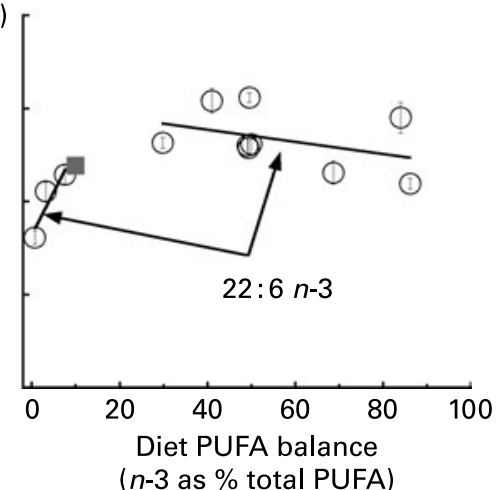

Fig. 2. Relationship between $20: 4 n-6$ and $22: 6 n-3$ content of rat muscle phospholipids and the amount of the respective $18-c a r b o n$ precursor fatty acid in the diet (graphs (a) and (c); $18: 2 n-6$ and 18:3n-3 content, respectively) and diet PUFA balance (graphs (b) and (d), respectively). In each graph, $\square$ represents value for initial rats (not used to determine line of best fit), while $\bigcirc$ represent data from experimental diets. Error bars show means with their standard errors, and if they are not visible they are smaller than symbol. See Table 4 for regression equations and statistical values.

PUFA balance (Table 4). Muscle $22: 5 n-6$ responded to diet composition to a greater extent than $22: 4 n-6$, but showed huge variability in response to diet $18: 2 n-6$ content (Table 4). The response to diet PUFA balance was biphasic, with a dramatic increase in muscle $22: 5 n-6$ as diet PUFA balance decreased below $10 \%$, while above this dietary PUFA balance muscle $22: 5 n-6$ remained relatively constant (Table 4).

The 20- and 22-carbon $n$-3 PUFA in muscle phospholipids were predominantly $22: 6 n-3$, with only small amounts of $20: 5 n-3$ and $22: 5 n-3$ present (Table 3). Small increases in both $20: 5 n-3$ and $22: 5 n-3$ were evident in muscle phospholipids with increases in both diet $18: 3 n-3$ content and PUFA balance (Table 4). The response of muscle $22: 6 n-3$ to diet composition was biphasic (Fig. 2(c) and (d)). Muscle $22: 6 n-3$ showed a steep relationship at low diet $18: 3 n-3$ contents $(<1 \mathrm{~g} / 100 \mathrm{~g})$, with a similar response to diet PUFA balance $<10 \%$ (Fig. 2(c) and (d)). Increasing the amount of diet $18: 3 n-3$ (above $2 \mathrm{~g} / 100 \mathrm{~g}$ ) led to a small decline in muscle 22:6n-3 (Fig. 2(c)). Levels of muscle $22: 6 n-3$ also showed a small decline as diet PUFA balance increased above $10 \%$ (Fig. 2(d)).

\section{Discussion}

Using the conformer/regulator concept ${ }^{(3)}$, we have quantified how responsive the fatty acid composition of rat muscle phospholipids differs from dietary fat profile. Slopes close to zero indicate membrane composition changes little in response to changes in diet fat profile (as with SFA, and less so with PUFA and MUFA), while increased slopes indicate increased responsiveness of membrane composition to dietary fat profile (e.g. n-3 PUFA, n-6 PUFA and PUFA balance). These results confirm that although the fatty acid composition of muscle phospholipids is largely a regulated parameter, the muscle composition of the essential dietary fatty acids, $n-3$ and $n-6$ PUFA, is most influenced by dietary composition. Furthermore, it is the dietary balance between these two types of PUFA that has the greatest influence on the fatty acid composition and, in some cases, that diet PUFA balance is the best predictor of membrane composition.

We have used PUFA balance ( $=n-3$ PUFA as percentage of total PUFA) because, as a 'proportion', it provides advantages over the more common use of 'ratios'. For example, using the $n-3: n-6$ ratio, when $n-6$ PUFA dominates in a mixture of the two, the value of the ratio will be between 0 and 1 ; however, when $n-3$ is the dominant PUFA in the mixture, the range of values will be $1-\infty$. Comparing (or averaging) ratios can be complicated, whereas the advantage of the PUFA balance is that the range of values are more even, ranging from 0 to $49 \%$ when $n-6$ PUFA dominate a mixture and 51-100\% when $n-3$ PUFA are dominant. Another advantage of using PUFA balance is that it can be more readily used to average out dietary intake. For example, if a subject consumes two meals both with the same total PUFA content, but one having 
a PUFA balance of $10 \%(n-3: n-6$ ratio $=0.11)$ and the other $90 \%(n-3: n-6$ ratio $=9 \cdot 0)$, then the average PUFA balance of these meals is $50 \%$, which is the actual situation of equal consumption of both PUFA types. However, averaging the $n-3: n-6$ ratios $(=4.6)$ does not give the same conclusion. These considerations suggest that PUFA balance has benefits in easier analysis of diets from the combinations of foods and meals.

An important feature of Fig. 2 is the greater strength of the linear relationships when 20- and 22-carbon PUFA are plotted against diet PUFA balance rather than when plotted against their 18-carbon precursors. This is most pronounced for muscle 20:4n-6, for example, the value of $R^{2} 0.35$ when plotted against diet $18: 2 n-6$ content compared with $R^{2} 0.83$ when plotted against diet PUFA balance. Thus, variation in diet PUFA balance can explain $83 \%$ of variation in muscle $20: 4 n-6$ content, while variation in diet $18: 2 n-6$ amount explains only $35 \%$ of muscle $20: 4 n-6$ variation. Similarly for $n$-3 PUFA, variation in diet PUFA balance explains $84 \%$ of variation in muscle $20: 5 n-3$ content and $80 \%$ of the variation in muscle $22: 5 n-3$ content, while the respective values for diet $18: 3 n-3$ content are only 58 and $48 \%$. This means that diet PUFA balance is a better predictor of muscle 20- and 22-carbon PUFA content than the amount of each essential fatty acid precursor in the diet.

Comparison of the effects on muscle composition of the diet level $v$. the balance between $n-3$ and $n-6$ PUFA can also be illustrated by a comparison of some individual diets. For example, diets 11 and 12 contained very different amounts of 18-carbon PUFA, but similar PUFA balances. Rats on these two diets had the same muscle phospholipid $n-3$ PUFA content even though there was $>3$-fold difference in the amount of 18-carbon $n$-3 PUFA in the diet. Muscle PUFA balance was not significantly different reflecting the similar PUFA balance of the two diets. Similarly, comparison of diets 8 and 9, which had very different total amounts of PUFA but the same PUFA balance, resulted in PUFA balance of muscle phospholipids that were not significantly different. Conversely, diets 1 and 9 had the same total PUFA content, but very different PUFA balances, and the PUFA balance of the muscle phospholipids was also very different. Furthermore, the muscle composition of the initial rats appears to conform to the relationships determined from the experimental rats, even though they were fed a diet with a lower fat content (13 v. $25 \%$ en). This also supports the importance of relative composition of fatty acids in the diet compared to absolute amount of fat in the diet in determining membrane fatty acid composition.

In the present study, rats were only provided with 18-carbon PUFA in the diet. This is a realistic natural diet for both rats and human subjects, with human subjects recorded to consume the majority of their $n-3$ PUFA in the form of $18: 3 n-3$ (approximately $1.3 \mathrm{~g} / \mathrm{d}$ ) and only a very small amount in the form of the 20- and 22-carbon n-3 PUFA (approximately $0 \cdot 1 \mathrm{~g} / \mathrm{d})^{(6)}$. There are a number of processes involved in the regulatory pathway between diet essential fatty acids and membrane PUFA composition. These include (i) conversion of the 18-carbon PUFA to longer chain PUFA by elongases and desaturases (both located in the endoplasmic reticulum), (ii) a peroxisomal processing step involving a single cycle of $\beta$-oxidation for highly unsaturated PUFA (i.e. $22: 6 n-3$ and $22: 5 n-6)$ and (iii) the deacylation-reacylation processes of membrane remodelling (involving phospholipases and acyltransferases).

An interaction between dietary $n-3$ PUFA and $n-6$ PUFA at the level of desaturases and elongases was first conclusively demonstrated by Mohrhauer \& Holman ${ }^{(7)}$. In their seminal study, the fatty acid composition of total lipids from liver, heart and depot fat was compared between rats provided a fat-free diet supplemented with daily oral doses of very small amounts of $18: 2 n-6$ and $18: 3 n-3$ as their sole source of dietary fat. They compared an impressive eighteen combinations of these two essential fatty acids. Intakes of $18: 2 n-6$ ranged from 0.07 to $0.73 \%$ en, while $18: 3 n-3$ intakes ranged from 0.05 to $1.92 \%$ en. Thus, the range of total PUFA intake was from 0.13 to $2.59 \%$ en (compared to $25 \%$ en from fat in the present study). They conclusively demonstrated that the conversion of $18: 2 n-6$ to $20: 4 n-6$ (from the combination of elongase, $\Delta 6$ and $\Delta 5$ desaturase activity) was inhibited by $18: 3 n-3$. We have calculated and plotted the PUFA balance of both diet and tissue lipids from their data (results not shown), and there is an impressive correlation coefficient of 0.98 for liver lipids and 0.92 for heart lipids. We have also plotted the relationship between diet PUFA balance and tissue $20: 4 n-6$ content from their data (results not shown), and the correlation coefficients were 0.95 and 0.88 for liver and heart lipids, respectively. These values are similar to the 0.91 correlation coefficient we found for the relationship between diet PUFA balance and 20:4n-6 content of muscle phospholipids, and suggest that diet PUFA balance may be a valuable predictor of membrane fatty acid composition irrespective of the amount of fat in the diet.

The final step in the biosynthesis of the highly polyunsaturated $22: 6 n-3$ and $22: 5 n-6$ involves the peroxisomes, where enzymes catalyse a single cycle of $\beta$-oxidation of 24 -carbon PUFA to produce these 22-carbon PUFA ${ }^{(8)}$. We know little about the regulation of their production, but the present results suggest it differs from their shorter chain less-unsaturated precursors. For example, at diet PUFA balances $>10 \%$, there appears to be no increase in $22: 6 n-3$ content of muscle phospholipids and negligible change in $22: 5 n-6$ content. However, at diet PUFA balance $<10 \%$, there is a dramatic increase in $22: 5 n-6$ and a decrease in $22: 6 n-3$. The level of $22: 5 n-6$ and $22: 6 n-3$ combined is constant irrespective of changes in diet PUFA balance. This suggests that the peroxisomal pathway may have a set capacity, which produces a steady total amount of these 22-carbon highly PUFA. It preferentially converts the available $n-3$ PUFA to $22: 6 n-3$, but will substitute $n$-6 PUFA to make $22: 5 n-6$ when there is inadequate $n-3$ PUFA for $22: 6 n-3$ production.

One of the most interesting findings was the greater predictability of $20: 4 n-6$ content of muscle phospholipids by diet 18-carbon PUFA balance compared with diet 18:2n-6 content. The incorporation of $20: 4 n-6$ into rat liver and brain phospholipids appears to primarily occur during the membrane remodelling (i.e. deacylation and reacylation) phase rather than during de novo synthesis of phospholipids $^{(9-11)}$; however, it is not definitely known whether this also applies to muscle phospholipids. Membrane lipid remodelling is performed by the combined action of phospholipases and acyltransferases. Lands ${ }^{(1)}$ has demonstrated that acyltransferases show no discriminating ability for the incorporation of $n-3$ and $n-6$ PUFA. This may be the 
mechanism responsible for the greater predictability of diet PUFA balance than diet $18: 2 n-6$ content on muscle $20: 4 n-6$.

To extrapolate these results in rodents to human subjects, one must take into account differences in lipid metabolism. Rodents have a higher capacity than human subjects in converting 18-carbon PUFA to their longer chain metabolites due to increased $\Delta 5$-desaturase activity ${ }^{(12)}$. Dietary $18: 3 n-3$ has been shown to increase mice plasma levels of $20: 5 n-3$ to a greater extent than in human subjects; however, the impact on plasma $22: 6 n-3$ and $20: 4 n-6$ was similar for mice and human subjects ${ }^{(13)}$. This study ${ }^{(13)}$ concluded that rodent diets including $n$-3 PUFA based on a percentage of total energy are most suitable for determining equivalent doses for human subjects. The range of percentage energy from dietary $18: 3 n-3$ in the present study was $0 \cdot 1-17 \cdot 3 \%$ en, with half of the experimental diets below the achievable human doses of $18: 3 n-3(<2 \%$ en). The diets with achievable human equivalent doses in the present study include diets with PUFA balances ranging from 0 to $50 \%$, which provides quite an extensive range. Furthermore, the total fat content of the diet is equivalent to the $20-35 \%$ recommended intake for human subjects ${ }^{(14-16)}$, so that some extrapolation to human conditions from this model is appropriate.

There are some implications of the present findings for the current human diet. Dietary intake studies have shown that the fat intake of the US averages a PUFA balance of approximately $9.5 \%^{(17)}$. If the present results in rats also apply to human subjects, an average PUFA balance of $9.5 \%$ in the modern human diet is of considerable concern, as it indicates there are huge numbers of people consuming a diet with a very low and likely inadequate PUFA balance. We have demonstrated dramatic reductions in $22: 6 n-3$ content and elevated 20:4n-6 in muscle phospholipids with low diet PUFA balance, which may have important implications as diet fat profile has been linked to the increasing incidence of metabolic and CVD as well as mental ill health ${ }^{(2,18-22)}$.

\section{Acknowledgements}

We gratefully acknowledge the technical assistance provided from Adam Zieba, Taleitha Atkins and Jessica Nealon. The present research was supported by a Discovery Project grant from the Australian Research Council. All authors disclose that there are no conflicts of interest. The study was conceived and planned by all three authors; the experiments were carried out by S. K. A. and the manuscript was written by all the three authors.

\section{References}

1. Lands WE, Inoue M, Sugiura Y, et al. (1982) Selective incorporation of polyunsaturated fatty acids into phosphatidylcholine by rat liver microsomes. J Biol Chem 257, 14968-14972.
2. Hulbert AJ, Turner N, Storlien LH, et al. (2005) Dietary fats and membrane function: implications for metabolism and disease. Biol Rev 80, 155-169.

3. Campbell NA, Reece JB \& Mitchell LG (1999) Biology, 5th ed. San Francisco, CA: Benjamin Cummings.

4. Folch J, Lees M \& Sloane Stanley GH (1957) A simple method for the isolation and purification of total lipids from animal tissues. J Biol Chem 226, 497-509.

5. Lepage G \& Roy CC (1986) Direct transesterification of all classes of lipids in a one-step reaction. J Lipid Res 27, $114-120$.

6. Arterburn LM, Hall EB \& Oken H (2006) Distribution, interconversion, and dose response of $n-3$ fatty acids in humans. Am J Clin Nutr 83, Suppl. 6, 1467S-1476S.

7. Mohrhauer H \& Holman RT (1963) Effect of linolenic acid upon the metabolism of linoleic acid. J Nutr 81, 67-74.

8. Sprecher H (2000) Metabolism of highly unsaturated $n-3$ and n-6 fatty acids. Biochim Biophys Acta 1486, 219-231.

9. van Golde LMG \& van den Bergh SG (1977) Liver. In Lipid Metabolism in Mammals, pp. 35-149 [F Snyder, editor]. New York: Plenum.

10. Holub BJ \& Kuksis A (1978) Metabolism of molecular species of diacylglycerophospholipids. Adv Lipid Res 16, $1-125$.

11. Schmid PC, Deli E \& Schmid HHO (1995) Generation and remodeling of phospholipid molecular species in rat hepatocytes. Arch Biochem Biophys 319, 168-176.

12. Willis AL (1981) Unanswered questions in EFA and PG research. Prog Lipid Res 20, 839-850.

13. Whelan J \& Jones L (2006) Allometric scaling: determining human equivalent doses for $n-3$ PUFA in rodent diets. FASEB $J$ 20, A550.

14. Dietary Guidelines for Australian Adults (2003) In National Health and Medical Research Council, 3rd ed. Canberra: AusInfo Government Info Bookshops.

15. Lichtenstein AH, Appel LJ, Brands M, et al. (2006) Diet and lifestyle recommendations revision 2006: a scientific statement from the American heart association nutrition committee. Circulation 114, 82-96.

16. Ryan-Harshman M \& Aldoori W (2006) New dietary reference intakes for macronutrients and fibre. Can Fam Phys 52, $177-179$

17. Ervin RB, Wright JD, Wang C, et al. (2004) Dietary intake of fats and fatty acids for the United States population: 19992000. In Advance Data from Vital and Health Statistics. Hyattsville, MD: National Center for Health Statistics.

18. Simopoulos AP (1999) Essential fatty acids in health and chronic disease. Am J Clin Nutr 70, 560S-569S.

19. Allport S (2006) The Queen Of Fats- Why omega-3s Were Removed From the Western Diet and What We Can Do To Replace Them. Los Angeles, CA: University of California Press.

20. Storlien L, Pan D, Kriketos A, et al. (1996) Skeletal muscle membrane lipids and insulin resistance. Lipids 31, S261-S265.

21. Vessby B (2000) Dietary fat and insulin action in humans. $\mathrm{Br} J$ Nutr 83, S91-S96.

22. Lands WE (2008) A critique of paradoxes in current advice on dietary lipids. Prog Lipid Res 47, 77-106. 